in THE LANCET of Oct. 28th (p. 1163), entitled "Virulent Acute Rheumatism with Extensive Parpura; Death from Cardiac Failure," and I strongly recommend all who are interested in the pathology and treatment of this malady to study carefully the history of this case and to pay particular attention to Dr. Poynton's remarks and inferences as to the condition found at the necropsy. I consider that this unfortunate case fully bears out what I have adrocated in THE LANCET and other journals during the past three years-viz, that "acute rheumatism" is primarily a joint affection due to some morbid material conveyed by the blood; that this poison, be it germ or ferment, gains admission to the body through the tonsil or other microbial trapdoor ; that the joint invasion is promptly followed by a form of acute arthritis and cellulitis with general toxæmia; and furthermore the infected joints serve as incubators where the poison is elaborated and poured into the circulation and through this latter agency conveyed to other articulations--the heart, the pericardium, and the pleura. Having such views I felt it necessary to suggest that the term "acute infective arthritis and cellulitis" be substituted for "acute rheumatiem," as the latter in reality was a vague expression with little or no etiological or pathological significance, and that the prefixes "gonorrhoeal," "pyæmic," "syphilitic," \&c., might be conveniently retained in order to differentiate the arthritis peculiar to each.

The next thing to find out was if there were any analogous affections, and if so, what treatment was most beneficial. Gonorrhcea and pyæmia bore resemblance in many respects, and I found that the only successful treatment for a gonorrhoeal or pyæmic joint was immediately to open, irrigate, and drain it. After an experience of 14 consecutive cases I can fearlessly state that similar treatment is a specific cure for acute infective arthritis and without in the least wishing to reflect on the treatment practised in Dr. Lees's case I cannot help thinking that if arthrotomy with drainage had been performed on both ankles and left knee on Jan. 17th the result might have been different. At the last meeting of the British Medical Association Sir R. Douglas Powell alluded to the possibility of this disease being an infective one, and needless to say any suggestion coming from such a distinguished authority deserves the serious consideration of specialists and practitioners alike. 1 have little doubt that before long salicylic acid and its derivatives will be superseded in the majority of cases by a scalpel and drainage tube, which to $\mathrm{my}$ mind is the rational treatment of the malady.

I am, Sirs, yours faithfully,

JoHN O'Coxor, M.A., M.D.T.C D.,

Senisr Medical Officer, British Hospital, Buenos Agres.

\section{THE MEDICAL SERVICE OF REMOTE SCOTLAND.}

To the Editors of THW LANOWT.

Sins,-A movement having been set on foot with the object of obtaining the privilege of a paid medical substitute for Scotch parish medical officers in outlying districts of the highlands and islands of Scotland during an annual holiday (such as has already been granted to dispensary officers in the remote districts of Ireland by the Local Government Board of Ireland), any medical officer concerned who desires to support a petition to the Local Government Board of Scotland for this object is requested to send in his name without delay to me.

I am, Sirs, yours faithfully

J. F. D. MACARA, M.B., C.M. Glasg.,

Dec. 12th, 1899 .

Parish Medical Officer, Durness, Sutheriandshire, N.B.

\section{THE TREATMENT OF INFLUENZA.}

To the Editors of THE LANCET.

SIBs, - It may be of service to some if I relate my experience of self-treatment for influenza, with a result of stopping the fever in from six to 10 hours. Part of my work at present is at the Military Hospital, Canterbury. There I have a ward which yesterday had 32 soldiers down with influenza. Finding yesterday midday that I was commencing the trouble I began with taking a half-drachm bi. carbonate of potassium powder every hour and as soon as I was able (2.30 P.M.) I began mercurial inunction. My plan is this, to smear the thirds of both feet between the toes and the heels with the present British Pharmacopoia unguentum hydrargyri and then to start walking. As I had to continue work until after six o'clock this was no trouble. I kept the socks and shoes (with a layer of thick brown paper over the inner soles to keep the shoes clean) on until bedtime, when I put on a clean pair of socks without removing the mercury, which was washed off this morning. My diet from lunch to bed-time was four basins of breadand-milk. My highest temperature was $1002^{\circ} \mathrm{F}$. at 7 P M. Altogether I took seven bicarbonate powders. At bed-time my temperature was only one point above the normal. When in bed $I$ took 10 grains of Dover's powder. I passed a very comfortable night, except for passing water every two or three hours, but I slept comfortably between times. This morning I am as fit as ever, at 8 o'clock eating a good meat breakfast, my temperature being below normal and no constipation.

Quite half-a-dozen times when attacked by influenza I have carried out this treatment with the same results-never being laid up. I am afraid it is hardly a method for private practice. I am, Sirs, yours, faithfully,

Dec. 6th, 1899. PUGIN Thornton.

\section{TREATMENT OF PUERPERAL SEPTIC AMIA BY PACKING THE BROAD LIGAMENTS WITH GAUZE.}

To the Editors of THE LANOET.

SIRS,-In watching severe cases of puerperal septicæmia it has occurred to me that it might be possible to partially isolate the uterus and so minimise absorption by opening and packing each broad ligament with gauze inserted either from the vagina or from above Poupart's ligament. I should prefer the latter. As the pregnant uterus during its enlargement draws up the broad ligaments and with them the peritoneum from the iliac fossæ no great difficulty ought to be experienced in entering the broad ligaments retro-peritoneally from above Poupart's ligaments. To simply curette and irrigate a puerperal uterus acutely infected throughout its whole thickness is next to useless and has resulted in the opposite extreme, bysterectomy, which is advocated by certain American gynæcologists. I can find no reference to this method of treating puerperal septicæmia but feel certain it must have been tried, and I am now writing with the object of ascertaining the views of those surgeons who have had experience of this treatment.

I am, Sirs, yours faithfully,

Plymouth, Dec. 18th, 1899. C. HAMILTON WHITEFORD.

\section{THE “ MEDICAL DIRECTORY" FOR 1900. To the Editors of THE LANCET.}

SIRS,-On Dec. 9th, by an extensive fire at our printers, the above work-which was on the eve of completion-was almost entirely destroyed, with the special type ased in composing it. We are taking the necessary steps to reprint, and hope, though it will be impossible to keep to the usual time of issue, to publish the volume early in February. Under the exceptional circumstances we trust that we may ask you to insert these few lines. We much regret the inconvenience from which our subscribers may suffer, but we feel confident that we may count upon their indulgence.

We are, Sirs, your obedient servants,

J. AND A. OHURCHILL.

Great Maslborough-street, W., Dec. 18th, 1899.

\section{DISCOMFORTS IN CONNEXION WITH THE JOURNEY TO AND FROM EGYPT.}

\section{To the Editors of THE LANCET.}

SIRs,-For many years past Egypt has been a favourite resort for invalids of a certain type, more particularly, perhaps, for those suffering from no very obvious signs of illness, who were nevertheless found by their medical advisers to have some point of natural or acquired weakness. The conditions to be found in Egypt were supposed to be an equable climate, abundant sunshine, and dry desert air. It has long been recognised that the evenings constituted a snurce of danger and all authoritative works on the 